\title{
Afectación de los bienestares en cuidadores de niños y adultos con enfermedad crónica
}

\section{Effects on welfare of children and adults with chronic disease caregivers}

Inna E. Flórez-Torres, Amparo Montalvo-Prieto, Arleth Herrera-Lían y Elizabeth Romero-Massa

Facultad de Enfermería, Universidad de Cartagena. Colombia. iflorezt@unicartagena.edu.co, amontalvop1@unicartagena.edu.co, aherreral@unicartagena.edu.co, eromerom@unicartagena.edu.co

Recibido 18 Junio 2010/Enviado para Modificación 12 Diciembre de 2010/Aceptado 24 diciembre 2010

\section{RESUMEN}

Objetivo Comparar los bienestares que integran la calidad de vida de los cuidadores familiares de niños y adultos con enfermedades crónicas, Cartagena, Colombia.

Método Estudio transversal realizado a 91 cuidadores de niños y 97 cuidadores de pacientes adultos de tres instituciones hospitalarias. Se empleó el instrumento de Ferrell y col. Para contrastar la afectación de los bienestares físico, psicológico, social y espiritual, se utilizó la prueba T de Student.

Resultados Predomina en ambos grupos la mujer como cuidadora, con edades entre 18 a 59 años. La proporción de cuidadores de niños con primaria incompleta es mayor que la de los adultos. Los bienestares psicológico y social están más afectados en los cuidadores de ACV, 48,7 frente a $50,5(T<0,05)$ y 21,8 frente a 19,2 $(T<0,05)$ respectivamente. En relación al bienestar espiritual los cuidadores de niños están significativamente más afectados que los de adultos 23,7 frente a $22.6(T<0,05)$. El bienestar físico no presenta diferencias significativas en los dos grupos 10,5 frente a 11,1 ( $T>0,05)$.

Conclusiones La experiencia de ser cuidador modifica de manera importante la percepción de la calidad de vida de las personas. Los cuidadores como fuente importante de cuidado informal requieren apoyo de los sistemas de salud y como parte de éste, de los profesionales de enfermería.

Palabras Clave: Calidad de vida, cuidadores, enfermedad crónica (fuente: DeCS, BIREME).

\section{ABSTRACT}

Objective Comparing the quality of caregivers' lives when they are related to child and adult patients suffering from chronic diseases (Cartagena, Colombia).

Method This cross-sectional study was carried out on 91 child and 97 adult patient caregivers in three hospitals located in Cartagena, Colombia. Ferrell et al.'s psychometric test was used for ascertaining social well-being. Student's t-test was 
used for testing the effect of care-giving on physical, psychological, social and spiritual well-being.

Results Females aged 18 to 59 predominated as caregivers in both groups. The percentage of child caregivers who had not completed primary school was higher than that of those looking after adults. Psychological and social welfare was most affected in stroke care-givers $(48.7$ cf $50.5(T<0.05)$ and 21.8 cf $19.2(T<0.05)$, respectively). Child caregivers were significantly more affected than adult caregivers regarding spiritual welfare $(23.7$ cf $22.6(\mathrm{~T}<0.05))$. There was no significant difference regarding physical wellbeing (10.5 cf 11.1 ( $(T>0.05))$.

Conclusions The experience of care-giving significantly modified people's perception of others' quality of life. Caregivers as an important source of informal care require health system support, particularly from nurses.

Key Words: Quality of life, caregiver, chronic disease (source: MeSH, NLM).

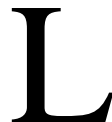

a Organización Panamericana de la Salud (OPS), señala, que las enfermedades crónicas no trasmisibles (ECNT) son la principal causa de muerte y discapacidad prematura en la gran mayoría de los países de América Latina y el Caribe. Tienen efectos devastadores para las personas, las familias y las comunidades, especialmente las poblaciones pobres, lo que se constituye en un obstáculo cada vez mayor para el desarrollo económico (1).

En Colombia, las ECNT son una causa importante de morbilidad y mortalidad y su tendencia es al aumento. Del total de muertes registradas durante 1990 al año 2005, la mortalidad por enfermedades crónicas se incrementó del $59 \%$ al $62,6 \%$ (2). La discapacidad guarda correspondencia con la situación mundial, aproximadamente 2640000 personas presentan alguna limitación permanente, lo cual equivale al 6,4 \% del total de la población colombiana (3).

Las ECNT se presentan en cualquier grupo de edad, mientras que en el adulto las causas más frecuentes se relacionan con la reducción de la mortalidad de las enfermedades crónicas no transmisibles, en el niño los factores genéticos inciden con mayor frecuencia en su producción. El $28 \%$ de todas las muertes se deben a los accidentes cerebro vasculares, constituyéndose en la segunda causa de años de vida potencialmente perdidos y la quinta de años potencialmente saludables perdidos entre quince y cuarenta y cuatro años de edad (4). Algunos autores documentan que en los países desarrollados, entre un 5 y un $10 \%$ de niños que no han cumplido los 18 años tienen situaciones de este tipo y muchos pueden llegar a tener una expectativa de vida más elevada si tienen al alcance la tecnología moderna, lo que implica nuevos retos en sus vidas y en la de sus cuidadores, bien sean éstos familiares o profesionales (5). 
La situación de los cuidadores y su calidad de vida (CV), ha despertado el interés por lo que se han generado diferentes tipos de estudio en los cuidadores. Estos estudios se han enfocado a grupos de edad específicos, llama la atención el estudio de Córdoba, Gómez y Verdugo, quienes plantean el surgimiento de un modelo de CV centrado en la familia; se abandona la perspectiva patológica, dándole confianza para desarrollar sus potencialidades y sus capacidades. La CV centrada en la familia, es influenciada significativamente por los factores personal y sociocultural, hacen referencia al impacto que tiene la pobreza en términos de menores oportunidades para todos los miembros de la familia. Infieren que aunque las madres sigan ejerciendo su rol de cuidadoras principales de sus hijos con discapacidad, esto no genera en ellas mayores niveles de estrés, ni sentimientos de frustración que incidan en su satisfacción con la calidad de vida (6).

Con relación al concepto de CV no se ha logrado un criterio claro, ya que este concepto comprende componentes subjetivos y objetivos. Los componentes subjetivos están relacionados con la percepción de las personas según sus propias características personales, necesidades, satisfacciones, aspiraciones, expectativas y escalas de valores de aspectos del entorno y de las condiciones globales de vida (7).

Merino (8), al comparar la CV de cuidadores familiares de niños en situación de enfermedad crónica hospitalizados, con la de los cuidadores usuarios de la consulta externa, determinó que ambos grupos presentaron comportamientos afectados en todos los bienestares de la calidad de vida, especialmente en el físico y el psicológico.

El cuidador familiar de una persona, adulto o niño, con enfermedad crónica ve afectada su CV y comprometido su núcleo familiar. Las implicaciones de la experiencia de cuidado de los cuidadores sobre su CV han sido documentadas, el cuidador se ve sometido a un estrés permanente por la limitación física, psicológica y cognitiva para realizar su labor, la alteración de la ejecución habitual de su rol, la de las interacciones sociales, la percepción de la falta de soporte social y la de apoyo de los sistemas de cuidado de la salud (8-12). No obstante, la $\mathrm{CV}$ de los cuidadores, no ha sido lo suficientemente abordada desde la perspectiva del contexto donde se desenvuelve el cuidado, la percepción del bienestar del cuidador de niños y la de adultos, sus diferencias o similitudes en la ejecución y consecuencias del rol, orientadas a dar respuestas desde la disciplina de enfermería, a las necesidades específicas y redefinir intervenciones de apoyo a quienes asumen una labor no reconocida por el sistema y las políticas de salud. 
La Organización Mundial de la Salud (OMS), define la CV como la percepción del individuo sobre su posición en la vida dentro del contexto cultural y el sistema de valores en el que vive y con respecto a sus metas, expectativas, normas y preocupaciones. Es un concepto extenso y complejo que engloba la salud física, el estado psicológico, el nivel de independencia, las relaciones sociales, las creencias personales y la relación con las características sobresalientes del entorno (13).

La CV se toma como el resultado de un proceso individual de valoración de muchas dimensiones que interactúan entre la persona y su entorno, abarca tanto condiciones de vida objetivas como subjetivas, dentro de las cuales se encuentran las normas sociales, las valoraciones personales y los factores afectivos $(7,12)$. La CV es parte esencial en el desarrollo de una sociedad; este concepto se ha relacionado con el consumo de bienes y se ha usado con frecuencia en diversos ámbitos como: salud, educación, economía, política, y en el mundo de los servicios en general (14).

Ferrel acepta la CV de los cuidadores como una evaluación subjetiva de los atributos bien sean positivos o negativos sobre la propia vida y consta de cuatro dominios que incluyen el bienestar físico, psicológico, social y espiritual (15). El bienestar físico, incluye la habilidad funcional, la salud física y los síntomas. El psicológico, incorpora la sensación del control, depresión, temor y felicidad. El social, se enfoca en los componentes de interrelación de la CV, incluidos las aflicciones familiares, aislamiento social, finanzas y función sexual. El bienestar espiritual los temas de significado y propósito de la vida, esperanza, incertidumbre y trascendencia (16).

El presente estudio, se propuso comparar los bienestares que integran la $\mathrm{CV}$ de los cuidadores familiares de niños y adultos con enfermedades crónicas, Cartagena, Colombia.

\section{MATERIALES Y METODOS}

Estudio transversal realizado durante el segundo semestre de 2008. La población estuvo constituida por los cuidadores familiares usuarios de la consulta externa y hospitalización de tres instituciones de tercer nivel de la ciudad de Cartagena. Se tomaron 188 cuidadores, 97 de adultos con secuelas de ACV y 91 de niños con cardiopatías congénitas complejas. Investigación de riesgo mínimo, conservó los parámetros éticos, aprobada por el comité de ética de la Universidad de Cartagena y de las instituciones participantes (17). Se aplicó la encuesta 
Caracterización de los Cuidadores, diseñada por el grupo de cuidado al paciente crónico de la Universidad Nacional de Colombia; la cual, consta de 13 preguntas cerradas y proporciona información sociodemográfica y de relación de cuidado, continuidad y tiempo, percepción de carga y de relación con la persona cuidada (18).

El instrumento empleado para medir la calidad de vida fue el propuesto por Ferrell y col. (1997) (15). Este instrumento valora la calidad de vida mediante 37 ítems que integran su significado en los bienestares: físico (5 ítems), psicológico (16 ítems), espiritual (9 ítems) y social ( 7 ítems). La evaluación de cada ítem se efectúa mediante una escala Likert de 1 a 4 . Para la interpretación de los resultados, los puntajes mínimos en la dimensión física, social y espiritual equivalen a una percepción positiva; los puntajes mínimos en la dimensión sicológica corresponden a una percepción negativa. En el proceso de validación el instrumento fue revisado y probado en un grupo de 219 cuidadores familiares de pacientes con cáncer en el período comprendido entre1994 a 1998. Los resultados de la validación mostraron una consistencia interna de 0.69 , medida con el estadístico alfa de Cronbach, y el análisis factorial confirmó cuatro dimensiones de calidad de vida como sub-escalas para el instrumento (19). En otro estudio, dirigido por Vargas y Pinto (20), realizado en pacientes conAlzheimer el instrumento presentó un alfa de Cronbach de 0.84 .

Los datos fueron procesados, se generaron estadísticas descriptivas sobre las características de los cuidadores. Para comparar los bienestares se utilizó la prueba T de Student. Se consideraron diferencias significativas aquellas que mostraron valores de probabilidad menores del $5 \%$.

\section{RESULTADOS}

El 95,6 \% de los cuidadores de los niños son mujeres en contraste con los cuidadores de los enfermos con ACV que es de $75 \%$. Las mujeres cuidadoras de niños son significativamente mayores $\left(X^{2}=15,3, \mathrm{p}<0,05\right)$. La distribución según edad es similar en los dos grupos $\left(X^{2}=5,76, p>0,05\right)$, es baja la proporción de cuidadores mayores de 60 años.

La escolaridad de los cuidadores de los 2 grupos, presentan diferencias relacionadas con los distintos niveles $\left(X^{2}=68,1, \mathrm{p}<0,05\right)$. La proporción de cuidadores de niños con primaria incompleta es mayor que los de adultos con $\mathrm{ACV}$, mientras la tercera parte de los cuidadores de secuelas de ACV han culminado secundaria, solo lo han hecho el $24 \%$ de niños. 
En relación al estado civil, la tendencia en los cuidadores de niños es que viven en unión libre ( 2 de cada 3 cuidadores), en los cuidadores de pacientes con ACV la distribución civil tiende a ser similar entre solteros, unión libre y casados. Hay diferencia entre los cuidadores de los dos grupos en torno al estado civil $\left(X^{2}\right.$ $=55,5 \mathrm{p}<0,05)$.

El tiempo de ser cuidador resulta no ser el mismo en los cuidadores de enfermos con ACV y los cuidadores de niños $\left(X^{2}=84,1 \mathrm{p}<0,05\right)$. El $71 \%$ de los cuidadores de niños lleva más de 19 meses en el cuidado de ellos en contraste con solo el 12 $\%$ de los cuidadores con ACV. Asimismo, los cuidadores de los enfermos con ACV dedican menos horas al cuidado que los de niños (Tabla 1).

El promedio de puntaje del bienestar físico de los cuidadores de adultos con secuelas de ACV es de 11,1 mientras que el de los cuidadores de niños con cardiopatías fue de 10,5. En ambos grupos, el bienestar físico de los cuidadores resulta estar igualmente afectado ( $\mathrm{T}>0,05)$ (Tabla 2).

El bienestar psicológico resulta afectado en los dos grupos de cuidadores, los de pacientes con ACV y los de niños (48,7 vs 50,5) (T>0,05), los primeros están significativamente afectados. De igual forma, los cuidadores de adultos con secuelas de ACV reportan un mayor promedio en cuanto al bienestar social, lo que demuestra que la afectación resulta ser significativamente mayor que la reportada por los cuidadores de niños $(T>0,05)$ (Tabla 2).

Contrario a lo anterior, los cuidadores de niños resultan estar significativamente más afectados que los de adultos con secuelas de ACV en relación al bienestar espiritual $(\mathrm{T}>0,05)($ Tabla 2$)$.

Tabla 1. Relación de cuidado de los cuidadores de niños y de personas con secuelas de ACV

\begin{tabular}{lcccc}
\hline \multicolumn{1}{c}{ Tiempo } & $\begin{array}{c}\text { Cuidadores } \\
\text { Niños }\end{array}$ & $\%$ & $\begin{array}{c}\text { Secuelas } \\
\text { de ACV }\end{array}$ & $\%$ \\
\hline 0 a 6 meses & 2 & 2,2 & 52 & 53,6 \\
7 a 18 meses & 24 & 26,4 & 33 & 34,0 \\
19 a 36 meses & 31 & 34,1 & 6 & 6,2 \\
Más de 37 meses & 34 & 37,4 & 6 & 6,2 \\
Horas dedicadas & & & & \\
Menos de 6 horas & 2 & 2,1 & 16 & 16,5 \\
7 a 12 horas & 24 & 24,7 & 33 & 34,0 \\
13 a 23 horas & 31 & 32,0 & 33 & 34,0 \\
24 horas & 34 & 35,1 & 15 & 15,5 \\
\hline \multicolumn{1}{c}{ Total } & 91 & 100,0 & 97 & 100,0 \\
\hline
\end{tabular}


Tabla 2. Contraste de medias de los bienestares de los cuidadores de personas con secuelas de ACV y de niños

\begin{tabular}{|c|c|c|c|c|c|c|}
\hline \multirow[t]{2}{*}{ Bienestar } & \multirow[t]{2}{*}{ Cuidadores } & \multirow[t]{2}{*}{ Promedio } & \multirow[t]{2}{*}{ Rango } & \multirow[t]{2}{*}{$\begin{array}{c}\text { Prob. } \\
\text { T }\end{array}$} & \multicolumn{2}{|c|}{$\begin{array}{c}95 \% \text { IC para la } \\
\text { diferencia de } \\
\text { medias }\end{array}$} \\
\hline & & & & & Inferior & Superior \\
\hline Físico & $\begin{array}{l}\text { Secuelas ACV } \\
\text { Niños }\end{array}$ & $\begin{array}{l}11,1 \\
10,5\end{array}$ & 5 a 20 & 0,149 & $-0,19$ & 1,27 \\
\hline Psicológico & $\begin{array}{l}\text { Secuelas ACV } \\
\text { Niños }\end{array}$ & $\begin{array}{l}48,7 \\
50,5\end{array}$ & 16 a 64 & 0,006 & $-3,1$ & $-0,5$ \\
\hline Social & $\begin{array}{l}\text { Secuelas ACV } \\
\text { Niños }\end{array}$ & $\begin{array}{l}21,8 \\
19,2\end{array}$ & 9 a 36 & 0,000 & 1,6 & 3,5 \\
\hline Espiritual & $\begin{array}{l}\text { Secuelas ACV } \\
\text { Niños }\end{array}$ & $\begin{array}{l}22,6 \\
23,7\end{array}$ & 7 a 28 & 0,005 & $-1,9$ & $=0,3$ \\
\hline
\end{tabular}

Cuidar sigue siendo un trabajo realizado fundamentalmente por mujeres. No obstante, los hombres cada vez se están vinculando más a esta labor. Los resultados del presente estudio así lo confirman, la mujer es la cuidadora principal de niños con cardiopatía $(95,6 \%)$ y adultos con ACV $(75,3 \%)$; similares resultados se reportan en Guatemala (21), donde la mayoría de los cuidadores familiares son mujeres, adultas, saben leer y escribir, en edad productiva (18 a 59 años), con una relación de hecho. En el presente estudio más de un $95 \%$ de los cuidadores se encuentran entre los 18 a 59 años; Blackburn (22), dice que las mujeres tienen más probabilidades de ser cuidadoras que los hombres y la proporción de responsabilidades de cuidados familiares aumenta con la edad, de manera que las comprendidas entre los 45-64 años son la mayoría.

Castillo et ál en un estudio de cuidadores de niños y adultos con cáncer, encontraron, que un alto porcentaje eran mujeres, en el grupo de cuidadores de niños eran sobre todo madres y abuelas y en los adultos hijos (as) y esposas. Lo cual se explica por el papel de cuidadora que se les ha asignado tradicionalmente a las mujeres en la cultura colombiana $(23,24)$.

Morales al igual que los resultados encontrados en cuanto al estado civil de los cuidadores (más del $65 \%$ casados y unión libre), expresa que alrededor del $75 \%$ de los cuidadoras están casadas o en unión libre (25); con relación al tiempo como cuidador, hay similitud con los datos encontrados en los cuidadores de niños, más de la mitad tienen 19 meses o más. Casi la totalidad de mujeres, por ser madres y amas de casa, se dedican a esa tarea 24 horas al día (25). Para Garcia-Calvente existen diferencias en las características del cuidado 
que prestan las mujeres y los hombres, tanto en el tipo de actividades, como en el tiempo dedicado a cuidar. Las mujeres asumen con mayor frecuencia los cuidados más pesados, cotidianos y que exigen una mayor dedicación. El cuidado, interfiere en la vida cotidiana de las mujeres mucho más que en los hombres, y las sitúa en mayor riesgo de padecer consecuencias negativas (26).

En los dos grupos de estudio, se encontró que los bienestares están afectados, el bienestar físico de los cuidadores de niños y adultos no presenta diferencias significativas en cuanto a la afectación, López Gil encontró evidencia acerca de la vulnerabilidad de los cuidadores a padecer problemas físicos y psíquicos, lo que determina la aparición del denominado síndrome del cuidador (27). En otros estudios $(28,29)$, las personas que asumen el cuidado de individuos que conviven con el virus del VIH-sida informan agotamiento físico y emocional, falta de conocimiento y recursos para lidiar con hijos adultos que padecen esta enfermedad, conflictos familiares, estigmatización social, discriminación, rechazo y apoyo.

Mateo- Rodríguez y otros (30) encontraron un alto porcentaje de cuidadores de personas con ECNT, con compromiso en su salud física; representando un mayor impacto los cuidadores de personas con enfermedades neurovegetativas, consecuencias negativas relacionadas con un detrimento y afectación. De la Cuesta expresa que el cuidado en la casa a un familiar no es sin costos; el cuidado a otro produce un desgaste físico y emocional, ampliamente documentado en la literatura bajo el término la carga del cuidado o el síndrome del cuidador (31).

El bienestar psicológico resulta afectado de distinta manera entre los dos grupos de cuidadores $(T>0,05)$, están más afectados los cuidadores de pacientes con ACV. Estudios reportan, aumento de procesos como ansiedad y depresión y síntomas como alteraciones del sueño, apatía o irritabilidad en el grupo de cuidadores respecto a la población de referencia, siendo incluso superiores a los encontrados $(26,27,32,33)$. Se ha relacionado además, un mayor deterioro en la esfera psíquica del cuidador con la prolongación del tiempo desempeñando este rol, la mayor dependencia de la persona atendida, la mayor sensación de sobrecarga y la menor presencia de apoyo social $(30,32)$.

Se evidencia mayor afectación del bienestar social en los cuidadores de adultos, al respecto Mateo-Rodríguez et al, encontraron alteraciones sociales como aislamiento, pérdida del tiempo libre; problemas laborales como absentismo, bajo rendimiento; conflictos familiares; problemas económicos, reducción de 
los ingresos o aumento de los gastos, o problemas legales (30). Ballesteros y col., en la valoración del funcionamiento social en cuidadores de niños; informaron poca afectación, excepto las preocupaciones económicas (34).

Hay evidencia significativa de mayor afectación del bienestar espiritual de los cuidadores de niños que de los adultos, $(\mathrm{T}>0,05)$. Pinto encontró, que la media en el bienestar espiritual de los cuidadores de niños fue de $(21,1 \%)$, por encima del promedio esperado $(17,5 \%)$, indicando mayor afectación. Un número apreciable de cuidadores $(29,9 \%)$, consideró insuficiente el nivel de apoyo espiritual que reciben por las prácticas religiosas y un 30,5\% reportó incertidumbre y duda relacionadas con el futuro del familiar bajo su cuidado (35). Sánchez reconoce la importancia del bienestar espiritual en el cuidado de las personas con y sin discapacidad. Para quienes viven situaciones de crisis, el bienestar espiritual puede significar un factor de crecimiento personal, un hecho trascendente que los acerca a muchas respuestas necesarias para dar sentido a la vida, a la cotidianidad, al dolor y al sufrimiento humano (36).

Los cuidadores familiares de ambos grupos se encuentran en la etapa de la adultez, desempeñan diferentes roles, representan una población económicamente activa, que por su condición ve limitada, y en consecuencia, con efectos devastadores por el estrés, al no sentir completamente control por las cosas que les suceden en la vida y estar alterada, la satisfacción, que les genera la misma.

Los resultados de este estudio comparativo son el primer aporte al conocimiento de enfermería en la ciudad, sobre el cuidado informal y sus implicaciones en la calidad de vida de los cuidadores.

Con el incremento de las enfermedades crónicas y la ausencia de políticas para los cuidadores familiares, la calidad de vida de este grupo continuará siendo afectada. Es preciso adelantar, al tiempo que se instauran cuidados para la salud de la persona en situación de cronicidad, acciones de cuidado para identificar en los cuidadores factores de riesgo para la salud, a fin de favorecer la experiencia positiva en el rol de cuidador y su CV.

Los resultados de este estudio se constituyen en fuente de análisis para nuevas propuestas de apoyo y generación de conocimiento propio para el cuidado de este grupo de personas 


\section{REFERENCIAS}

1. Organización Panamericana de la Salud [Internet]. Resumen: estrategia regional y Plan de Acción para un enfoque integrado sobre la prevención y el control de las enfermedades crónicas. 2007. Disponible en: http://www.paho.org/spanish/ad/dpc/nc/reg-stratcncds.pdf. Consultado: agosto de 2009.

2. Lucumi D, Gutiérrez A, Moreno J, Gómez L, Lagos N, Rosero M, et al. Planeación local para enfrentar el desafío de las enfermedades crónicas en Pasto, Colombia. Rev. Salud Pública 2008; 10(2):343-351.

3. Censo 2005. Resultado sobre limitaciones permanentes [Internet]. Bogotá: Departamento Administrativo Nacional de Estadística. Disponible en: http://discapacidadcolombia.com/ modules.php?name=News\&file=article\&sid=1268. Consultado: Agosto de 2009.

4. Guía de práctica clínica para las enfermedades cerebrovasculares 2007; 5(1) Especial [Internet]. Disponible en: http://www.medisur.sld.cu/index.php/medisur/article/view/246/3708 Consultado: Abril de 2008.

5. United Nations. Clasificación Internacional de Deficiencias, Discapacidades y Minusvalías (CIDDM). Boletín sobre los discapacitados; 1999 (2) [Internet]. Disponible en: http:// www.un.org/esa/socdev/enable/dpbe19992c.htm Consultado: Febrero de 2007.

6. Córdoba-Andrade L, Gómez-Benito J, Verdugo-Alonso MA. Calidad de vida familiar en personas con discapacidad: un análisis comparativo. Universitas Psychologica. 2008; 7(2): 369-383.

7. Zea M, López ME, Valencia CM, Soto JA, Aguirre DC, Lopera, et al. Autovaloración de calidad de vida y envejecimiento en adultos con riesgo de Alzheimer. Invest educ enferm., 2008; XXVI (1): 24-35.

8. Merino S. Calidad de vida de los cuidadores familiares que cuidan niños en situación de enfermedad crónica. Av. Enferm., 2004; 22 (1): 39-46.

9. Álvarez B. Comparación de la calidad de vida de cuidadores familiares de personas que viven con el $\mathrm{VIH} /$ sida y reciben terapia antirretroviral con la calidad de vida de los cuidadores familiares de personas con el $\mathrm{VIH} /$ sida y no reciben terapia antirretroviral de Honduras. Av. Enferm. 2004; 22 (2): 6-18.

10. Barrera L. La cronicidad y el cuidado familiar, un problema durante la niñez. Av. Enferm., 2004; 22(1): 47-53.

11. Moreno ME, Nader A, López C. Relación entre las características de los cuidadores familiares de pacientes con discapacidad y la percepción de su estado de salud. Av Enferm. 2004; XXII (1): 27-38.

12. Escuredo B. Cuidadores informales, necesidades y ayudas. Rol de enfermería 2001; 24 (3): 23-29.

13. Group Whoqol. Study protocol for the World Health Organization project to develop a Quality of Life assessment instrument (WHOQOL). Qual Life Res 1993; 2: 153-159.

14. Tovar RL, Puerto H. Promoción de la salud. Lineamientos para la promoción de la salud y la estrategia promocional de la salud. En: Asociación Colombiana de Facultades de Enfermería. Directrices para la enseñanza de enfermería en la educación superior. Bogotá: Gráficas Ducal; 2006: 117-136.

15. Ferrell B. La calidad de las vidas: 1525 voces del cáncer. ONS/Bristol-Myers. 1996; 23 (6): 153-159.

16. Ersek M, Ferrell B, Dow k, Melancon C. Quality of life in woman with ovarian cancer. Western Journal of nursing research, 1997; 19(3): 334-350.

17. Colombia. Ministerio de Salud. Resolución 008430 de 1993, Octubre 1993. Por el cual se establecen las normas científicas técnicas y administrativas para la investigación en salud. Bogotá D.C.; 1993.

18. Barrera L, Pinto N, Sánchez B. Caracterización de los cuidadores familiares en América Latina. En: Barrera L, Pinto N, Sánchez B, Carrillo GM, Chaparro L. Cuidando a los Cuidadores Familiares de personas con enfermedad crónica. Universidad Nacional de Colombia, 2010: 45-56. 
19. Ferrell B, Ervin K, Smith S, Marek T, Melancon C. Family perspectives of ovarian cancer. Cancer practice. 2002: 10(6): 269-276.

20. Vargas LM, Pinto N. Calidad de vida del cuidador familiar y dependencia del paciente con Alzheimer. Av. enferm. 2010: 28(1): 116-128. En: http://www.enfermeria.unal.edu.co/ revista/articulos/xxviii1_12.pdf. Consultado: Octubre de 2010.

21. Morales ON. Calidad de vida de los cuidadores familiares de personas que viven en situación de enfermedad crónica discapacitante. En: Enfermería en Guatemala. Revista anual. Año 1. Época 1. Septiembre 2007: 9-19.

22. Blackburn C, Read J, Hughes N. Carers and the digital divide: Factors affecting internet use among carers. Health and Social Care in the Community. 2005; 13(3): 201-210.

23. Castillo E, Chesla CA, Echeverri G, Tascón EC, Charry M, Chicangana J A, et al. Satisfacción de los familiares cuidadores con la atención en salud dada a adultos y niños con cáncer [Internet]. Colombia Médica, 2005. 36(3). Disponible en: http:// colombiamedica.univalle.edu.co/Vol36No3Supl1/PDF/cm36n3s1a5.pdf. Consultado: Julio de 2009.

24. Díaz L. Cómo perciben los cuidadores la situación crónica de enfermedad del niño. En: El arte y la ciencia del cuidado. Universidad Nacional de Colombia, Facultad de Enfermería. Bogotá: Unibiblos; 2002: 227-229.

25. Morales ON. Efectos del programa Cuidando al cuidador, en la mejora de la habilidad de cuidado. Av. Enferm. 2008; XXVI (2): 27-34.

26. García-Calvente M, Mateo-Rodríguez I, Maroto-Navarro G. El impacto de cuidar en la salud y la calidad de vida de las mujeres [Internet]. Gaceta sanitaria. Disponible en: http:// scielo.isciii.es/scielo.php Consultado: abril de 2008.

27. López- Gil MJ, Orueta-Sánchez R, Gómez-Caro S, Sánchez-Oropesa A, Carmona de la Morena J, Alonso-Moreno FJ. El rol del cuidador de personas dependientes y sus repercusiones sobre su calidad de vida y su salud. Rev Clin. Med. Fam. 2009; 2 (7): 332-339.

28. Hanzeliková A [Internet]. El sida y las personas mayores. Gerokomos 2006; 17(2): 33-39. Disponible en: http://scielo.isciii.es/scielo.php?script=sci_arttext\&pid=S1134928X2006000200004 Consultado: Marzo de 2008.

29. Montalvo-Prieto A, Flórez-Torres I. Caracteristicas de los cuidadores de personas en situación de cronicidad. Cartagena (Colombia): Un estudio comparativo. Barranquilla. Rev Salud Uninorte. 2008; 24 (2): 181-190.

30. Mateo-Rodríguez I, Millán-Carrasco A, García-Calvente MM, Gutiérrez-Cuadra P, GonzaloJiménez E, López-Fernández LA [Internet]. Cuidadores familiares de personas con enfermedad neurodegenerativa: perfil, aportaciones e impacto de cuidar. Aten Primaria. 2000; 26: 25-34. Disponible en: http://www.doyma.es/revistas/ ctl_servlet?_f=7064\&ip=190.9.213.221\&articuloid=11295\&revistaid=27. Consultado: Mayo de 2009.

31. De la Cuesta-Benjumea C. Familia y cuidados a pacientes crónicos: el papel de la enfermera en el cuidado familiar. Index Enferm. 2001; 10(34): 20-26.

32. Badia X, Lara N, Roset M. Calidad de vida, tiempo de dedicación y carga percibida por el cuidador principal informal del enfermo de Alzheimer. Aten Primaria 2004; 34:170-177.

33. López O, Lorenzo A, Santiago P. Morbilidad en cuidadores de pacientes confinados en su domicilio. Aten Primaria 1999; 24: 404-410.

34. Ballesteros BP, Novoa MM, Muñoz L, Suárez F, Zarante I [Internet]. Calidad de vida en familias con niños menores de dos años afectados por malformaciones congénitas: Perspectiva del cuidador principal. Univ. Psicol.; 2006 (3): 457-473. Disponible en: http:// www.scielo.org.co/pdf/rups/v5n3/v5n3a03.pdf. Consultado: Agosto de 2009.

35. Pinto N. Bienestar espiritual de los cuidadores familiares de niños que viven enfermedad crónica. Investigación en Enferm. 2007; 9 (1): 19-35.

36. Sánchez-Herrera B. Bienestar espiritual en personas con y sin discapacidad. Aquichan. 2009; 9 (1): 8-22. 